the same time with splendid results, both in gout and rheumatoid arthritis. I have had two cases of acute gout to treat by this method and the relief was immediate. The agonising shoots of pain disappeared after the first application and the slight tenderness which remained soon vanished after a second. Needless to say, the cedema rapidly disappeared after the first. The immediate relief is one of the most marvellous things in medicine. A case of monarticular arthritis, probably rheumatoid, who had been treated by other methods for a couple of years with little success, and who could only walk with the aid of a stick when he came, was so far recovered after two and a half months as to be able to take a 30 -mile walk and return to his duty as a policeman.

How far can a rheumatoid arthritis or gout be cured? It is, of course, at present impossible to answer this question, but it does not seem likely that we can cause the absorption of bony deposits, and we certainly cannot get rid of bony ankylosis. With regard to the cavities in bone so commonly found, however, the case is different, and one hopes in time to get these filled up with normal bone. It is yet too early to say anything definite, especially as the patients get so much better that they return to work and cease attendance at the hospital.

Zinc has been more used for iontophoresis than any other ion. In rodent ulcer the zinc ions seem to have a selective action on the growth, causing the skin to heal over and the nodules to disappear. In America it has been used for large epitheliomas; a general anæsthetic is given and a very large current used. In a case of fungating epithelioma which was ionised with zinc I have found considerable fibrosis in the ionised parts. The growth was, however, too big to be dealt with even by heroic measures.

In rodent ulcer care must be taken to get rid of all the nodules. The healthy skin is protected by jaconet or oilsilk and a current of three milliampères per square centimetre passed for from 25 to 30 minutes. At the end of the application the ionised part will be a glistening white colour. A certain amount of inflammation follows which disappears in five or six days, the ulcer rapidly heals, and the nodules shrink away. If any nodules are left the application must be repeated. In the majority of cases I prefer massive doses of $x$ rays for this disease becanse the are painless; they search out nodules of the disease which cannot be seen or felt, and if a carefully measured full dose is given every 17 to 22 days there seems no danger of producing telangiectases as in the old method of frequent small doses. The scar in either case is very good. Employing much smaller doses of zinc I have had most excellent results in chronic ulcers of the leg, especially the varicose variety the only other treatment used is a dressing of vaseline and a properly applied calico bandage. The patient goes about his or her work as usual. I have been able to cure cases of many years' standing and cases treated by every other means. The most satisfactory thing is the immense relief to pain afforded after the first few hours, which persists for many days.

Zinc ions appear to be useful for all similar conditions such as erosion of the cervix, fissure of the anus, mucous colitis, and chronic sinuses. Copper ions have been used for sinuses with success, but I have not found them of much use in ulcers. They have been recommended for ringworm, and in the one case in which I used them $I$ obtained a cure, and there was no recurrence six months afterwards. Hartigan has also reported successes in lupus erythematosus with copper. Magnesium ions are very useful for the ordinary multiple warts that one sees on the hands, though on some varieties they appear to have no effect.

Such, then, are the uses to which iontophoresis has already been put and they are both numerous and varied. There is a great field for its use in other skin diseases and also in affections of joints and muscles. For instance, I should think that sulphur ions (from, say, sulphide of soda) would be useful in many chronic skin affections but have had no opportunity to try it. Further, it is the only non-vital method we have for extracting substances from the tissues, all the other methods aiming at stimulating the excretory giands. There has been of late years a growing prejudice against the use of drugs. Some people prefer to administer an extra dose of the same poison which is causing the symptoms, and others to fill the diseased part up with effete products by obstructing the circulation. The pendulum will, however, swing back and drugs will again become fashionable, but in many cases in this more efficient form, and the old local applications will be a thing of the past.

The uses of iontophoresis are certain to be very considerably extended in a few years, so much so that the battery will become as common as the stethoscope and every student will be taught its uses. At present, however, the specialists must still hold the field, and it is for us to work out the applications of the treatment and by establishing the pharmacology of ions to lift their use from the realms of empiricism and place them on a solid pedestal of fact from which they can never be dethroned.

Welbeck-street, W.

\section{A CASE OF PRIMARY OVARIAN ACTINOMYCOSIS. ${ }^{1}$}

BY FRANK E. TAYLOR, M.D. LOND., M.R.C.P. LOND., F.R.C.S. ENG., D.P.H. CAMB.,

GYNACOLOGIST TO THE HAMPSTEAD GENERAL AND NORTH-WHSI LONDON HOSPITALS; OBS'LETRIC REGISTRAR AND TUTOR, THE MIDDLASFX HOSPITAL; LATE PATHOLOGIST TO THE CIIELSEA HOSPITAL FOR WOMLA, IONDOX;

$$
\text { AND }
$$

WELBY E. FISHER, F.R.C.S. ENG.,

LATE RESIDENT MEDICAL OFFICER, CHELSEA HOSPITAL FOR

$$
\text { WOMEN, LOXDOX. }
$$

ACTINomycosis is one of the rarer forms of infection of the human tissues, whilst actinomycosis of the ovary constitutes one of the curiosities of gynæcology, only six cases of this condition being on record. In none of these has the ovary been the primary seat of infection but has been infected by extension of the disease originating either in the intestines or in the vagina, uterus, and Fallopian tubes. In the case now recorded the ovary was the primary seat of the disease : it is, therefore, a unique case.

A woman, aged 34 years, single, was admitted into Chelsea Hospital for Women, under the care of Dr. W. H. Fenton, on June 24th, 1908, complaining of abdominal pain. Menstruation commenced at the age of 11 years and was associated with severe pain in the lower abdomen but was quite regular until the age of 17 years, when the patient's health failed; she became thin and drowsy and menstruation was completely arrested for 18 months. Her health then improved, she became fatter and stronger, and menstruation returned but was now very irregular and very profuse, the periods lasting from 10 to 14 days and requiring two or three dozen diapers, the intervals varying from five days to three months. About October, 1904, the patient being then 30 years of age abdominal pain was first noticed. It was felt in the right hypogastrium, being very severe and throbbing in character, and was aggravated by the recumbent posture and relieved by walking. About this time she also began to sweat profusely at nights and this continued until admission. After suffering in this way for about six weeks the patient saw Dr. Fenton, who found the right ovary enlarged; he diagnosed an ovarian cyst and recommended admission into hospital with a view to operation. This was declined. The pain and discomfort continued intermittently for 12 months, the patient meanwhile continuing at work. She then began to suffer excruciating pain on micturition, and for two months passed blood and pus in great quantity in the urine, which also contained small fleshy masses of about the size of grapes. A London practitioner was then consulted. He informed the patient that she had a displaced womb and ordered medicine and hot douches and inserted a ring pessary which had to be removed in three days on account of the pain caused thereby. In a couple of months, the pus having then disappeared from the urine, the pessary was reinserted. It now gave relief and was worn for three months. There was no vaginal discharge. The temperature was never taken. The bowels as a rule were constipated. In February, 1908, the patient had a very severe flooding and a fainting attack, and the right side from the hip to the toes was disabled by cramp which was present continuously night and day for 14 days. The same medical man was again consulted and he reinserted the pessary. There was now a thick yellow vaginal dis. charge, and although the ring was removed in a few days

1 A paper read at a meeting of the Gynecological and Obstetrical Section of the Royal Society of Medicine on March 11th, 1909. 
the discharge continued. As the patient's condition was gradually becoming worse she again consulted Dr. Fenton, who, diagnosing a suppurating ovarian cyst, ordered her immediate admission into hospital.

On admission in June, 1908, the patient complained of severe hypogastric pains which were relieved by the fourhourly application of hot fomentations. On pelvic examination the cervix was felt to lie low in the vagina. It was eroded and soft and seemed to expand widely into a hard tumour of about the size of a fœtal head. The tumour was of an even consistence, was free from tenderness, and accurately filled the pelvis. It was incarcerated below the pelvic brim, above which it could neither be felt nor percussed. By manipulation in the genu-pectoral position it was pushed slightly into the abdomen without distress to the patient. The abdominal and thoracic organs and the nervous systems showed no abnormality. The urine was acid and of specific gravity 1010 ; it contained no albumin or sugar. On admission the temperature was $102^{\circ} \mathrm{F}$., the pulse was 102 , and the respirations were 28 per minute. On the evening of the same day the temperature rose to $103^{\circ}$ and the pulse to 120 . On the next morning the temperature had fallen to normal, to rise in the evening to $102^{\circ}$; and on the third morning, that of operation, had fallen to normal and remained so after removal of the tumour. (See Chart.)

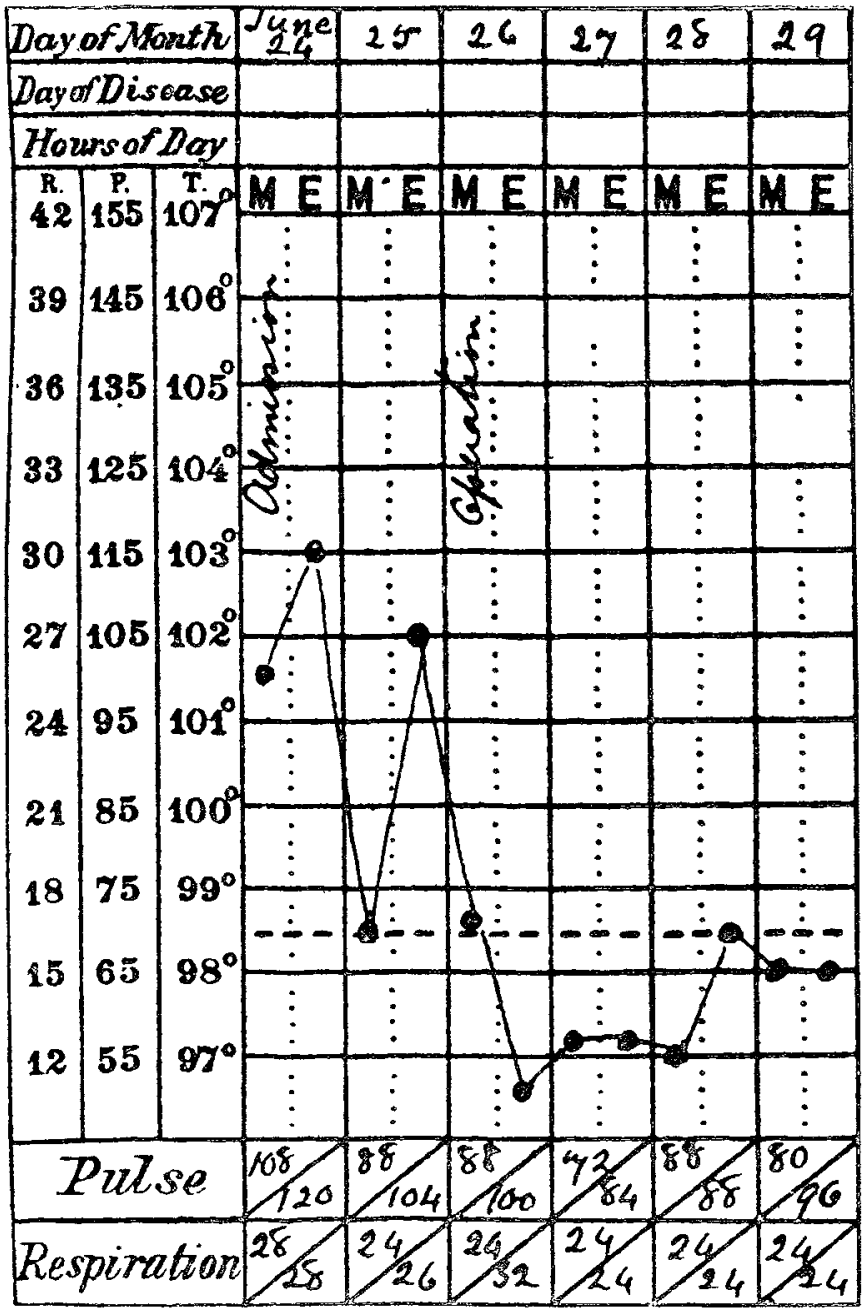

On June '26th Dr. Fenton performed a median coeliotomy. The right broad ligament was found to lie in front of, and to cover, a rounded tumour of about the size of a foetal head. The tumour was covered with purulent and caseous material which on removal of the mass adhered to the tumour bed. Both Fallopian tubes were occluded and bilateral salpingooöphorectomy was performed. The abdominal wound was closed in three layers and a large rubber drainage-tube was inserted.

There was marked shock after the operation, the temperature falling to $96^{\circ}$, with excessive sweating and cold clammy skin, and the temperature did not return to normal until the third day. There was much discharge through the drainagetube, which was changed for a smaller one next day and removed on the fourth day, a good deal of thin serous discharge escaping on removal of the tube. The bowels were opened by enemata on the third and by aperients ori the fourth day. There was no abdominal distension. Urine was passed by catheter in fair amount.

On June 30 th, that is, the fourth day after operation, at 3 A.M., after a good night's sleep, the patient passed 12 ounces of urine naturally, the act of micturition being accompanied by acute abdominal pain. The temperature was $97^{\circ}$, the pulse was 114 , and the respirations were 32 per minute. She became very ill during the day and by the evening was much collapsed. The temperature had fallen to $96^{\circ}$, the pulse being 132 and very feeble, and the respirations 36 . The skin was cold and clammy, perspiration was profuse, thirst was great, and the bowels were opened frequently. The abdomen was a little distended, there was no discharge from the wound, and no localised collection or swelling could be detected. On examination of the thorax a to-and-fro rub was heard at the base of the heart. It alternated in intensity with the respirations and indicated pericarditis. Vigorous treatment was carried out to counteract the heart failurecold compresses were applied to the cardiac area, tinctura strophanthi, mx., was given every four hours, and brandy, $\xi$ ss., every two hours. In the evening large Spencer Wells forceps were passed through the drainage opening deep amongst the intestinal coils and a tube was reinserted. An abundant flow of flaky sero-purulent discharge escaped. The condition became worse during the night, the pulse being very feeble and rapid, approaching 200 per minute. Brown fluid was frequently vomited and the bowels were opened several times. Champagne was given occasionally; saline injections per rectum were not well retained. Two half-pints of normal saline solution were infused into the left median cephalic vein, the patient being insensitive to the incision. The pupils were widely dilated. On the next day, July 1st, the patient vomited green fluid frequently. Liquor strychninæ, in v., was administered followed by in iii. every three hours. The pulse was 132 and very feeble during the day. As the vomiting persisted the stomach was washed out in the evening. During the night the patient slept four hours, passing urine and stool in her sleep.

After this there was a very gradual improvement and the salines and stimulants were gradually withdrawn. Occasional vomiting continued and only fluids could be retained for some weeks. Milk was badly borne. Sweating continued to be a marked feature. On July 7 th the tube was withdrawn as the discharge had become very slight. On the 9th there was marked urgency of micturition with dysuria and the urine became cloudy and offensive; the cystitis continued for ten days. On the 16th, on vaginal examination the uterus was felt to be fixed to the right pelvic wall by an indurated mass. A probe passed four inches along the original drainage-tube track.

On July 23rd the patient was discharged from hospital. She was confined to bed at home for a week and then went to the Convalescent Home at St. Leonards. After a week at the Convalescent Home the lower abdomen became swollen and hard. Hot fomentations were applied and a large abscess burst, pints of pus escaping. As the sinus persisted with copious discharge she was readmitted into the Chelsea Hospital on Oct. 3rd. In general health she was greatly improved, but the abdominal wound had broken down, there being several openings discharging pus, the lowest of which led deeply towards the pelvic cavity. Shortly after admission urine escaped from this opening. Cystoscopy showed a small ragged opening at the fundus of the bladder.

On Oct. 18th an anæsthetic was given and the abdominal scar was incised and granulomatous material was removed by a sharp spoon. No micro-organism could be found in this material and inoculated culture media remained sterile. A catheter was inserted and retained for ten days. On the day following its removal urine again escaped, associated with a brief febrile attack, the temperature running up to $103^{\circ}$ and the pulse to 120 per minute. The discharge of urine soon ceased and the wound healed except along the original drainage-tube track, where a sinus remained discharging yellow pus. From cultures of this pus the bacillus coli was obtained. Since early in December the patient has been taking iodides in large doses and her general condition has gradually improved. The sinus has not quite healed, a spot of discharge showing on the gauze dressing each 24 hours.

The structures removed at operation consisted of the uterine appendages of both sides. The left appendages showed evidences of chronic inflammation in a thickened and indurated Fallopian tube, with closure of the abdominal 
ostium, the ovary and mesosalpinx being normal. There was no sign of actinomycosis in the left appendages, either on macroscopic or on microscopic examination. The right appendages also showed a thickened and indurated Fallopian tube attached to the broad ligament which was infiltrated with soft friable tissue from which a solid tumour representing the ovary had become detached. This separate tumour consisted of an irregularly ovoid solid mass with a tuberose and in places worm-eaten-like surface. It was of a uniformly greyish-yellow colour and measured, after hardening in formalin, $2 \frac{3}{4}$ by $2 \frac{3}{4}$ by $3 \frac{3}{4}$ inches, being 10 inches in circumference. It weighed 73 ounces. On cutting across the mass uniform yellow pus was seen to exude from numerous points, the cut section presenting a regular honeycomblike structure, while towards one pole of the tumour a larger abscess cavity of about the size of a walnut was present. Nodules characteristic of actinomycotic pus were not observed with the naked eye. Films of the pus were made and stained with methylene blue and degenerate pus cells and granular detritus were seen but no microorganisms of any sort. Agar tubes were also inoculated with the pus and incubated at $37^{\circ} \mathrm{O}$. but remained sterile.

A portion was excised for microscopic examination and the tumour was then hardened whole in formalin solution. Sections were stained with hæmatoxylin and eosin. The ovary was transformed into a mass of loose connective tissue with marked small, round-celled infiltration, scattered irregularly throughout which were small foci of suppuration. No trace of ovarian structure was left. The cellular infiltration of the connective-tissue stroma consisted mainly of lymphocytes. A few giant cells were observed scattered through the connective tissue, but these were not surrounded by zones of epithelioid cells and lymphocytes as in typical tubercles. The giant cells were multinuclear, the nuclei being massed around the periphery of the cells, and in some of the nuclei typical somatic mitotes could be seen.

Further sections were subsequently taken from less degenerate parts of the tumour and stained for bacillus tuberculosis by the Ziehl-Neelsen's method, but this organism was not found, nor did the streptothrical mycelium retain the red colour of the carbol-fuchsin, which showed that it was not an acidfast streptothrix. Sections were also stained by Gram's method and counter-stained with eosin. The scattered purulent collections were well seen by this method as pink areas, throughout which small masses of a streptothrix retaining Gram's stain were found. These masses were composed of a close felt-work of mycelium in which the filaments were somewhat granular in appearance, their structure being best seen at the periphery of the mycelial masses. No club forms were observed. These appearances are all typical of sections of actinomycosis as affecting human tissues.

The source of the streptothrix and its mode of ingress to the body must in our case remain somewhat doubtful. Except for rare and brief holidays the patient had lived in London as cook in doctors' families for the last 16 years and prior to that time was at school in Bristol, but was brought into contact with hay, straw, and corn, the usual sources of actinomycosis, in 1903 and 1904, when the house in which she was engaged had a stable near the back door, the patient going into the stable regularly three or four times a day to fetch fuel and sometimes to feed the horse. We may possibly here find the explanation as to how the disease was contracted, since the symptoms date from 1904.

Since the ovary occupies a secluded position in the closed abdominal cavity, it can only be infected either by the direct extension of disease from an affected contiguous organ or by the blood-stream. As in this case there was no evidence of disease in any adjacent organ the streptothrix must have reached the ovary by way of the blood-stream, the mode of entry into the body being, we suggest, some cryptogenic focus-e.g., the tonsil, through which it is well known micro-organisms may pass into the blood-stream without producing any local lesion. It may further be pointed out that the ovary provides a peculiarly fertile soil for the growth of infective micro-organisms and of detached carcinoma cells, as evidenced by the frequent occurrence of large ovarian growths secondary to mammary and intestinal carcinomata. It can thus be readily understood that the streptothrix may have been deposited in the ovary by the blood-stream and so have given rise to a primary ovarian actinomycosis.

We are indebted to the kindness of Dr, Fenton for permission to publish this case,

\section{A METHOD OF TREATING FRACTURE OF THE CLAVICLE.}

\section{BY FRANK ROMER, M.R.C.S. ENG., L.R.C.P. LOND.}

I HAVE had the opportunity of treating a number of fractures of the clavicle by a method which has given me such satisfactory results that I venture to draw attention to the advantages which it possesses. A brief description of the injury may serve to illustrate the raison d'etre of the method advocated.

The usual condition, when the injury has been caused by indirect violence, is for the inner fragment to be kept in position by the rhomboid ligament so as to cause a prominence at the seat of fracture, the outer being displaced downwards and inwards, whilst the shoulder drops forwards and downwards. This displacement is caused by the action of the serratus magnus and pectoralis minor muscles, unrestrained by the stay of a sound clavicle, drawing the scapula forwards, and is not, as frequently stated, due to the weight of the upper extremity. This can be shown by the fact that merely supporting the arm so as to remove the weight causes no alteration in the deformity. On the other hand, if the shoulder be drawn back or the patient laid flat on his baok so that the scapula is pushed backwards reduction is effected. From this it appears that the best way of keeping the fragments in good position and preventing the return of the deformity is to render as far as possible the scapula immobile.

In the method which I employ, therefore, no attempt is made to confine the arm to the side, and, once the scapula is fixed, the movements of the arm need no more restriction than can be obtained by an ordinary sling. The technique of the method is thus described: "Three strips of firm adhesive plaster, each an inch and a half in width, should be applied, from a point immediately above the nipple to a point below the angle of the scapula. The middle strip should cover the seat of the fracture and should be first applied; the lateral ones, slightly overlapping it, should extend about an inch and a half on either side. Each strip should first be made to adhere strongly in front, and, while it is supported and fixed by the fingers of one hand, should be carried over the shoulder by the other, with steady pressure, and made to adhere as it goes." In addition to these strips I have found it advisable to apply another which encircles the shoulder-joint, one end being brought diagonally across the scapula to below its angle. To prevent chafing or the plaster adhering to the hairs a thin layer of wool should be placed in the axilla. The strapping once applied, the patient can dress in the ordinary way, the arm of the injured side being supported by a sling.

For the first two days use of the arm should be restricted to underhand movements, though one rarely finds much desire on the part of a patient to do more. As soon as possible the services of a skilled rubber should be obtained and the injured parts daily massaged for at least a week. I should mention that the rubbing can be effectively performed over the plaster. At the end of this time the sling can usually be discarded and the patient can be safely encouraged to take the arm into more general use. The strapping should be renewed at the end of the first four days and again from time to time as occasion demands. By the end of a fortnight all movements are as a rule possible, but the lifting of heavy weights should be avoided. The parts should be kept supported by strapping for at least three weeks, even though no pain be experienced on movement. I quote the following cases:-

CASE 1. - A man, a flat-race jockey, fell during a race and fractured his right clavicle. He was attended to on the course and the fracture was put up in the orthodox method. He was seen by me on the day of the accident and the shoulder was strapped and rubbing was ordered. He kept his arm in a sling for ten days. He rode and won a race 17 days after the injury without ill effects, but he had some pain as the horse "yawed" at the post. The shoulder was strapped once afterwards but he continued to ride regularly.

CASE 2.-..The patient, a flat-race jockey, sustained a complete fracture of the left clavicle through the fall of his horse in a race. $\mathrm{He}$ was seen six days after treatinent by the orthodox method. He was in considerable pain and distortion was still present at the junction of 